\title{
Theory for Diffusion-Limited Oscillating Chemical Reactions
}

\author{
Harmen J. Bussemaker \\ Institute for Physical Science and Technology \\ University of Maryland \\ College Park, Maryland 20742 \\ Ricardo Brito \\ Facultad de Ciencias Físicas \\ Universidad Complutense \\ 28040 Madrid, Spain \\ (J. Stat. Phys. 87, 1165-1178 (1997).)
}

\begin{abstract}
A kinetic description of lattice-gas automaton models for reaction-diffusion systems is presented. It provides corrections to the mean-field rate equations in the diffusion-limited regime. When applied to the two-species Maginu model, the theory gives an excellent quantitative prediction of the effect of slow diffusion on the periodic oscillations of the average concentrations in a spatially homogeneous state.
\end{abstract}

Keywords: Reaction-diffusion, lattice-gas automata, non-detailed balance, Hopf bifurcation. 


\section{INTRODUCTION}

In this paper we describe how a relatively simple theory quantitatively explains the deviations from mean-field behavior that occur in diffusion-limited chemical reactions. The modeling of chemical reactions in spatially extended systems is an interesting application of a class of microscopic models called "lattice-gas automata" [1]. Space, velocity, and time are all discrete in such models, which simplifies implementation on computers as well as theoretical analysis. Lattice-gas automata (LGA) provide a flexible tool for studying the various phenomena resulting from the interplay between reaction and diffusion [2].

Here we will not be concerned with chemical pattern formation, but instead we will consider a spatially extended two-species model exhibiting coupled periodic oscillations of the concentrations of both species in a spatially homogeneous state. If the reactions are slow compared to the diffusion, then mean-field or Boltzmann theory equations give an excellent description of the reaction kinetics. This is the so-called reaction-limited regime. In the opposite diffusion-limited case however, when the diffusion is slow compared to the reactions, there is no time to equilibrate after a reaction before another reaction occurs. Consequently, equal-time correlations will be present that invalidate the Stosszahlansatz or molecular chaos assumption used to derive the Boltzmann equation. Therefore in the diffusion-limited regime the behavior of the system is seriously modified.

A condition that guarantees the absence of correlations in the equilibrium state of latticegas automata is the so-called detailed balance (DB) condition. Reactive LGA's in the diffusion-limited regime violate DB. A systematic theory for LGA's violating DB has recently been developed by Ernst and coworkers [3, [1]. In the present paper we apply this theory to calculate corrections to the Boltzmann equation. A similar method has been developed by Boghosian and Taylor [5]:6].

The organization of this paper is as follows. In section $\mathbb{1}$ we define the model used. We present the ring kinetic theory in section III, and compare it with computer simulations in section $\mathbb{I \nabla}$. We end with a discussion in section $\nabla$.

\section{THE MODEL}

\section{A. Reactive Lattice-Gas Automaton}

In a lattice gas automaton particles live on a regular lattice, $\mathcal{L}$, so that their positions can only take a a limited set of values corresponding to the nodes of the lattice. The velocities are also restricted, and must be equal to unit vectors oriented along the the links connecting the neighboring nodes. We denote this set by $\left\{\mathbf{c}_{i} ; 1 \leq i \leq b\right\}$ where $b$ is the coordination number of the lattice. The square lattice, with $b=4$, will be used in this paper as it has sufficient symmetry to properly describe the diffusive problem that we are considering. We further impose an exclusion principle requiring that no more that one particle can be at the same node with the same velocity. As a consequence there can be at most $b$ particles

per node, i.e., one per link. The state of the LGA is fully described by a set of boolean occupation numbers $\left\{s_{i}(\mathbf{r}) ; 1 \leq i \leq b, \mathbf{r} \in \mathcal{L}\right\}$, where $s_{i}(\mathbf{r})$ equals 1 if there is a particle at node $\mathbf{r}$ with velocity $\mathbf{c}_{i}$ and 0 otherwise. 
For multi-species models with $m$ types of reactants, such as the Maginu model where $m=2$, we have to introduce different types of particles. The exclusion principle has to be modified in order to allow for the coexistence of several species. We adopt the coupledlattice model described in [7]. In this approach particles of different types live on separate lattices, and only interact when a chemical reaction occurs. The exclusion principle is applied independently to each lattice. However, for the sake of compactness in the mathematical derivations, we can extend the former set of occupation numbers $s_{i}(\mathbf{r})$ to a new set $\left\{s_{i}(\mathbf{r}) ; 1 \leq\right.$ $i \leq m b, \mathbf{r} \in \mathcal{L}\}$, in such a way that channels $1 \leq i \leq b$ are reserved for particles of species 1 , channels $b+1 \leq i \leq 2 b$ for species 2 , etc. The number of particles of type $p$ is given by

$$
\alpha_{p}(\mathbf{r})=\sum_{i=1+(p-1) b}^{p b} s_{i}(\mathbf{r}) .
$$

A time evolution step is the composition of two substeps, defined as follows. First, at each node independently a reactive collision takes place, during which a pre-reaction state $s(\mathbf{r}) \equiv\left\{s_{i}(\mathbf{r}), 1 \leq i \leq m b\right\}$ is replaced by a post-reaction state $\sigma(\mathbf{r})$ in a stochastic process governed by a set of transition probabilities $A_{s \sigma}$. The reactive collision is followed by a propagation step, during which all particles are moved to neighboring nodes $\mathbf{r}+\mathbf{c}_{i}$ in the direction of their velocities.

Let us describe the reactive collision step in detail. The chemical reaction we want to simulate is described by

$$
\alpha_{1} X_{1}+\alpha_{2} X_{2}+\ldots+\alpha_{m} X_{m} \longrightarrow \beta_{1} X_{1}+\beta_{2} X_{2}+\ldots+\beta_{m} X_{m}
$$

and occurs at a rate $P(\boldsymbol{\alpha}, \boldsymbol{\beta})$, where $\boldsymbol{\alpha}=\left(\alpha_{1}, \alpha_{2}, \ldots\right)$ and $\boldsymbol{\beta}=\left(\beta_{1}, \beta_{2}, \ldots\right)$ specify the number of particles before and after reaction, and $X_{p}$ represents species $p$. The outcome of the chemical reaction only depends on the number of particles of each species, $\left\{\alpha_{p}(s) ; 1 \leq\right.$ $p \leq m\}$, present at the node before the reaction, not on the velocity distribution. After the reaction, the $\beta_{p}$ particles of each species are randomly redistributed over the $b$ available velocity directions (this random redistribution models the diffusion process), which can be done in $b ! /\left(\beta_{p}\right) !\left(b-\beta_{p}\right)$ ! ways for species $p$. Thus, the transition probability from precollision state $s$ to postcollision state $\sigma$ is given by

$$
A_{s \sigma}=\left[\prod_{p=1}^{m} \frac{\left(\beta_{p}(\sigma)\right) !\left(b-\beta_{p}(\sigma)\right) !}{b !}\right] P(\boldsymbol{\alpha}, \boldsymbol{\beta}) .
$$

Note that the normalization $\sum_{\sigma} A_{s \sigma}=1$ follows from the normalization $\sum_{\beta} P(\boldsymbol{\alpha}, \boldsymbol{\beta})=1$.

\section{B. Maginu Model}

The Maginu model [8] is a two species model that exhibits a variety of behavior. It is described by the following equations for the concentrations $x$ and $y$ of the two species [2]:

$$
\begin{aligned}
& \frac{\partial x}{\partial t}=x-x^{3} / 3-y+D_{x} \nabla^{2} x \\
& \frac{\partial y}{\partial t}=(x-k y) / c+D_{y} \nabla^{2} y
\end{aligned}
$$


with $c>0$ and $0<k<1$. The constants $D_{x}$ and $D_{y}$ are the diffusion coefficients for the two species respectively. Depending on the parameters, the model can exhibit Turing structures (when $D_{x}$ is very different from $D_{y}$ ) as well as periodic behavior. Here we will solely be interested in the case $D_{x}=D_{y}$, where the system develops a stable limit cycle in a homogeneous state. This limit cycle shrinks as the chemical reaction rate increases.

The Maginu model as defined by Eq. (雨) is not directly useful since the concentrations can become negative, and therefore cannot be simulated with an LGA [7]. This problem is however easily solved by using the linear transformation

$$
\begin{aligned}
& x=\frac{1}{2}+x / \sqrt{12(1+k) / k}, \\
& y=\frac{1}{2}+y k / \sqrt{12(1+k) / k}
\end{aligned}
$$

where $x$ and $y$ are the concentrations of the two species $X$ and $Y$ that we will study.

Next we have to determine a set of reaction rates $P(\boldsymbol{\alpha}, \boldsymbol{\beta})$ for the LGA that gives rise to the macroscopic behavior defined by Eqs. (4) and (5). The matrix $P(\boldsymbol{\alpha}, \boldsymbol{\beta})$ is needed in numerical simulations as well as in the theory presented in the next section. In Ref. [2] a method for constructing $P(\boldsymbol{\alpha}, \boldsymbol{\beta})$ has been extensively discussed, and we will not give the details here. We will however, adopt the rules of Ref. [7], where the number of particles is only allowed to change by \pm 1 during the reaction. The matrix $P(\boldsymbol{\alpha}, \boldsymbol{\beta})$ is then uniquely specified.

An important point in the definition of the collision rules is the introduction of a time scaling parameter, $s$, which allows us to control whether the system is in the reactionlimited or in the diffusion-limited regime (see Ref. [7] for details). For large values of $s$ we have $P(\boldsymbol{\alpha}, \boldsymbol{\beta}) \simeq \delta(\boldsymbol{\alpha}, \boldsymbol{\beta})$ (where $\delta$ is the Kronecker delta): chemical reactions occur at a very slow rate. This is the diffusion-limited regime, where diffusion is able to maintain the homogeneity in the system, and where Eqs. (4) and (5) are meaningful, as the conditions for their derivation are fulfilled. On the other hand, for small values of $s$ chemical reactions occur at a much faster rate, and diffusion is no longer able to maintain spatial homogeneity. This is the reaction-limited regime. In the next section we present a theory that explains the behavior of the system throughout both regimes.

\section{RING KINETIC THEORY}

In mean-field or Boltzmann approximation all correlations between occupation numbers are neglected, and the state of the system is completely specified by the average occupation numbers,

$$
f_{i}(\mathbf{r}, t)=\left\langle s_{i}(\mathbf{r}, t)\right\rangle .
$$

The time evolution of $f_{i}(\mathbf{r}, t)$ is given by the nonlinear Boltzmann equation,

$$
f_{i}(\mathbf{r}, t+1)=f_{i}(\mathbf{r}, t)+I_{i}[f(\mathbf{r}, t)] .
$$

The nonlinear collision operator is defined as

$$
I_{i}[f]=\sum_{s, \sigma}\left(\sigma_{i}-s_{i}\right) A_{s \sigma} F(s) \equiv\left\langle\sigma_{i}-s_{i}\right\rangle_{F} .
$$


We have introduced $\langle\cdots\rangle_{F}$ as an average that assumes that the precollision state is factorized over all channels, so that the probability to find a state $s$ is given by

$$
F(s)=\prod_{i} f_{i}^{s_{i}}\left(1-f_{i}\right)^{1-s_{i}} .
$$

In this approximation, where $F(s)$ is given by Eq. (9), and the transition rates $A_{s \sigma}$ are those of the Maginu model, the nonlinear Boltzmann equation (17) is equivalent to the mean-field rate equations (4) and (5).

To go beyond the mean-field approximation we consider the pair correlation function,

$$
\mathcal{C}_{i j}\left(\mathbf{r}-\mathbf{r}^{\prime}, t\right)=\left\langle\delta s_{i}(\mathbf{r}, t) \delta s_{j}\left(\mathbf{r}^{\prime}, t\right)\right\rangle
$$

Here we have assumed that the system is translationally invariant. The fluctuations are defined as $\delta s_{i}=s_{i}-f_{i}$. A special role is played by the on-node correlations $\mathcal{C}_{i j}(\mathbf{0}, t)$; by definition the diagonal elements vanish: $\mathcal{C}_{i i}(\mathbf{0}, t) \equiv 0$. We neglect all triplet and higher order correlations. In a spatially homogeneous system, where $f_{i}(\mathbf{r}, t)=f_{i}(t)$, the time evolution of $f_{i}(t)$ is then described by the generalized Boltzmann equation,

$$
f_{i}(t+1)-f_{i}(t)=I_{i}[f(t)]+\sum_{k<l} I_{i, k l}^{\prime}[f(t)] \mathcal{C}_{k l}(t) .
$$

Here the operator $I^{\prime}$ describes corrections to the Boltzmann collision term $I$. It is defined by

$$
I_{i, k l}^{\prime}[f]=\frac{\partial^{2} I_{i}[f]}{\partial f_{k} \partial f_{l}}=\left\langle\left(\sigma_{i}-s_{i}\right) \frac{\delta s_{k} \delta s_{l}}{g_{k} g_{l}}\right\rangle_{F},
$$

where $g_{i}=\left\langle\left(\delta s_{i}\right)^{2}\right\rangle=f_{i}\left(1-f_{i}\right)$ is the single channel fluctuation.

In order to have a complete theory we must provide a time evolution equation for $\mathcal{C}_{i j}(\mathbf{r}, t)$. To derive this equation we will make the important assumption that the average occupations change slowly in time. In fact, as far as the evolution of $\mathcal{C}_{i j}(\mathbf{r}, t)$ is concerned, we will assume that no chemical reactions occur at all, so that the model is purely diffusive. Under this assumption, the average occupations in equilibrium are given by

$$
\begin{array}{ll}
f_{i}^{\mathrm{eq}}=x & (i=1,2,3,4) \\
f_{i}^{\text {eq }}=y & (i=5,6,7,8),
\end{array}
$$

where $x$ and $y$ are the average concentrations of species $X$ and $Y$, respectively. When $f_{i}(\mathbf{r}, t)$ is close to equilibrium, the approach to equilibrium is given by the linearized Boltzmann equation $\left(\delta f_{i}=f_{i}-f_{i}^{\mathrm{eq}}\right)$,

$$
\delta f_{i}\left(\mathbf{r}+\mathbf{c}_{\mathbf{i}}, t+1\right)=\sum_{j}(\mathbb{1}+\Omega)_{i j} \delta f_{j}(\mathbf{r}, t)
$$

where $\mathbb{1}_{i j}=\delta_{i j}$ and the linearized Boltzmann operator is defined by

$$
\Omega_{i j}=\frac{\partial I_{i}[f]}{\partial f_{j}}=\left\langle\left(\sigma_{i}-s_{i}\right) \frac{\delta s_{j}}{g_{j}}\right\rangle_{F} .
$$


Under the assumption of slow reactions we have

$$
(\mathbb{1}+\Omega)=\frac{1}{4}\left(\begin{array}{llllllll}
1 & 1 & 1 & 1 & 0 & 0 & 0 & 0 \\
1 & 1 & 1 & 1 & 0 & 0 & 0 & 0 \\
1 & 1 & 1 & 1 & 0 & 0 & 0 & 0 \\
1 & 1 & 1 & 1 & 0 & 0 & 0 & 0 \\
0 & 0 & 0 & 0 & 1 & 1 & 1 & 1 \\
0 & 0 & 0 & 0 & 1 & 1 & 1 & 1 \\
0 & 0 & 0 & 0 & 1 & 1 & 1 & 1 \\
0 & 0 & 0 & 0 & 1 & 1 & 1 & 1
\end{array}\right) .
$$

It is natural to assume that fluctuations $\delta s_{i}(\mathbf{r}, t)$ will decay to equilibrium in a manner similar to $\delta f_{i}(\mathbf{r}, t)$, i.e.,

$$
\delta s_{i}\left(\mathbf{r}+\mathbf{c}_{i}, t+1\right)=\sum_{j}(\mathbb{1}+\Omega)_{i j} \delta s_{j}(\mathbf{r}, t) .
$$

However, two fluctuations at the same node will be correlated after collision, even if before collision the distribution is completely factorized. This is a consequence of the violation of detailed balance [3]. The generation of on-node postcollision correlations is quantified by

$$
\Omega_{i j}^{2,0}[f(t)]=\left\langle\delta \sigma_{i}(\mathbf{r}, t) \delta \sigma_{j}(\mathbf{r}, t)\right\rangle_{F} .
$$

This expression vanishes in the non-reactive limit $s \rightarrow \infty$. The presence of on-node correlations $\mathcal{C}_{i j}(\mathbf{0}, t)$ before collision gives rise to corrections to $\Omega_{i j}^{2,0}[f(t)]$, and the full postcollision source term is given by (see Ref. [3])

$$
B_{i j}(t)=\Omega_{i j}^{2,0}[f(t)]+\mathcal{C}_{i j}(\mathbf{0}, t)+\sum_{k, l} \Omega_{i j, k l}^{2,2}[f(t)] \mathcal{C}_{k l}(\mathbf{0}, t),
$$

where $\Omega_{i j, k l}^{2,2}[f]=\partial^{2} \Omega_{i j}^{2,0}[f] / \partial f_{k} \partial f_{l}$. Combining Eqs. (17) and (19) with the definition of $\mathcal{C}_{i j}(\mathbf{r}, t)$ in Eq. (10) we obtain the ring kinetic equation

$$
\mathcal{C}_{i j}\left(\mathbf{r}+\mathbf{c}_{i}-\mathbf{c}_{j}, t+1\right)=\left(1-\delta_{\mathbf{r}, \mathbf{0}}\right) \sum_{k, l}(\mathbb{1}+\Omega)_{i k}(\mathbb{1}+\Omega)_{j l} \mathcal{C}_{k l}(\mathbf{r}, t)+\delta_{\mathbf{r}, \mathbf{0}} B_{i j}[f(t)] .
$$

This equation has been derived in a more systematic fashion in Ref. [3].

The physical interpretation of Eq. (20) is as follows. Two fluctuations on the same node $\mathbf{r}$ that are correlated after collision at time $t_{0}$, will be propagated to neighboring nodes $\mathbf{r}+\mathbf{c}_{i}$ and $\mathbf{r}+\mathbf{c}_{j}$. Due to the collision with other particles at these nodes the correlation will be scattered to all directions as described by $(\mathbb{1}+\Omega)$. Thus both fluctuations branch into many different paths. At time $t_{0}+\tau$ the weight of each path is given by the same factor $(1 / 4)^{\tau}$. If two correlated paths end at the same node - a so-called "ring"-collision - they give rise to on-node precollision correlations, $\mathcal{C}\left(\mathbf{0}, t_{0}+\tau\right) \sim(1 / 4)^{2 \tau} B\left(t_{0}\right)$, that change the time evolution of the average occupations according to Eq. (11). The actual value of $\mathcal{C}_{i j}(\mathbf{r}, t)$ is a superposition of "ring" contributions from source terms at all earlier times, although the dominant contribution comes from the last few time steps.

The fact that Eq. (20) is linear in $\mathcal{C}$ allows us to write 


$$
\mathcal{C}_{i j}(\mathbf{0}, t)=\sum_{t^{\prime}=0}^{t-1} \sum_{k, l} K_{i j, k l}\left(t-t^{\prime}\right) B_{k l}\left(t^{\prime}\right) .
$$

Here $K_{i j, k l}\left(t-t^{\prime}\right)$ is a memory kernel which does not depend on any of the model parameters - although it does depend on the system size $L$ - and thus can be constructed once and for all using Eq. (20). This can be done in an efficient manner by exploiting the rotation and reflection symmetry of $K_{i j, k l}\left(t-t^{\prime}\right)$.

After an initial fast decay, the memory function decays algebraically for large $t$, as $K_{i j, k l}(t) \propto t^{-\alpha}$ with $\alpha \simeq 1.2$ for $L=256$. When the ring kinetic theory is evaluated numerically, on time scales on the order of $10^{3}$ time steps this slow decay leads to the buildup of pair correlations that are much larger that what is observed in simulations. This excess of correlations would be corrected if we include higher order correlations that are not taken into account by the the present form of the ring kinetic theory. Therefore it is desirable to cut off the memory kernel for large times, i.e., to set $K_{i j, k l}(t) \equiv 0$ for $t>t_{\text {cutoff }}$. It is natural to choose the cut-off equal to the time it takes to travel across the the system: $t_{\text {cutoff }}=L$.

By rewriting Eq. (20) in Fourier representation, it can be interpreted in terms of modes at different wavevectors $\mathbf{q}$ (see Ref. [3]). When no reactions occur, the diffusive modes around $\mathbf{q}=\mathbf{0}$ and the (spurious) staggered modes around $\mathbf{q}=(\pi, \pi)$ play a special role, since they correspond to conserved densities. However, in the presence of reactive collisions there are no conserved densities, and all modes are in principle equally important in determining the size of the correlations.

In the next section we will compare theoretical predictions with the results of computer simulations. Numerically, the theory of this section is evaluated as follows. At time $t=0$ we set $f_{i}(0)=x_{0}$ for $1 \leq i \leq 4, f_{i}(0)=y_{0}$ for $5 \leq i \leq 8$, and $\mathcal{C}_{i j}(\mathbf{r}, 0)=0$. To perform a time evolution step from time $t$ to time $t+1$, we first use $f(t)$ to calculate the nonlinear Boltzmann operator $I_{i}[f(t)]$ and the correction term $I_{i, k l}^{\prime}[f(t)]$. Together with the on-node correlations $\mathcal{C}_{i j}(\mathbf{0}, t)$ we then use these operators to calculate $f_{i}(t+1)$ with the help of Eq. (11). To calculate the evolution of the pair correlation function we use $f_{i}(t)$ and $\mathcal{C}_{i j}(\mathbf{0}, t)$ to evaluate the source term $B_{i j}(t)$ in Eq. (19) and then obtain $\mathcal{C}_{i j}(\mathbf{r}, t+1)$ with the help of Eq. (20). Iteration of the above procedure yields the set $\{[x(t), y(t)] ; t \geq 0\}$ defining a trajectory in the $x-y$ concentration plane. For large times, either a fixed point or a limit cycle is reached.

\section{COMPARISON WITH SIMULATIONS}

Our simulations were carried out on a $256 \times 256$ square lattice. The parameters used in the simulations were $k=0.9$ and $c=2$, i.e., identical to those used in Ref. [7]. At $t=0$ the system was prepared in an uncorrelated homogeneous state, with average concentrations $x_{0}=y_{0}=0.6$. Then we performed the time evolution of the LGA, according to section III. The initial time steps were discarded, as the system needs some time to build up the correlations that will eventually produce the shrinking of the limit cycle. Once the correlations have been created we record the spatially averaged concentration of both species. The scale parameter $s$ was varied between $s=2$ and $s=20$.

In Fig. 1 the dashed line denotes the limit cycle as it is obtained from the mean-field theory defined by Eqs. (田) and (5), assuming that the concentration of both species are homogeneous and the term $\nabla^{2}$ can be neglected. For relatively large values of the time 
scaling parameter $s$ we expect mean-field theory to be accurate. This is confirmed by the simulation data for $s=20$, shown as a gray band in Fig. 1, which are reasonably close to the mean-field prediction. The width of the gray band corresponds to the fluctuations in the spatially averaged concentrations that occur due to the finite system size.

When $s$ is decreased correlations become important (measurements show that correlations are typically 10 times larger for $s=4$ than for $s=20$ ) and the diffusion process is not able to keep the system homogeneous. As a consequence, different regions in the system become desynchronized to a certain degree, and the contribution to the average concentration of one region is partially canceled out by out-of-phase contributions from other regions. This produces a shrinking of the limit cycle in Fig. 1, as is shown by the simulation data for $s=6$ and $s=4$ in Fig. 1. The effect is stronger for smaller $s$.

It is clear from Fig. 1 1 that mean-field theory completely fails for the smaller values of $s$. The solid black lines in Fig. 1 1 represent the limit cycle as it is predicted by the ring kinetic theory of section $\mathbb{1 T I}$. For the values $s=20$ and $s=6$ shown in Fig. 11 our theory gives an excellent quantitative prediction of the shrinking of the limit cycle. For $s=4$ there are deviations due to higher order effects that are not taken into account.

Analysis of the ring kinetic theory shows that as $s$ is further decreased, the limit cycle shrinks continually, until at $s \simeq 3$ there is an inverse Hopf bifurcation from a limit cycle to a fixed point. This bifurcation corresponds to a desynchronization transition, where the coherence between different regions is lost completely. It should be noted that this transition is of a different character than the Hopf bifurcation that occurs at the mean-field level as a function of the model parameters $k$ and $c$.

Let us consider the case $s=2$ in some detail. Here $s$ is close to the smallest possible value (see Ref. [7]) and the fluctuations caused by the chemical reactions are strongest here. Diffusion is not fast enough to keep the system homogeneous except at very small scales. The ring kinetic theory for $s=2$ predicts a fixed point located at $x=y=1 / 2$. Simulations for a system of linear size $L=256$ reveal that the average concentrations fluctuate around the point $x=y=1 / 2$ in a irregular fashion, and in a range between 0.47 and 0.53 . In order to assess whether the result of the simulations for $s=2$ corresponds to a fixed point or to a limit cycle, we compared numerical simulations for three different system sizes: $L=32$, $L=256$, and $L=1024$. The concentration of species $X$ versus time is plotted in Fig. 2 . The vertical scale in all three plots is the same. Clearly, the amplitude of the oscillation decreases with the system size. In the $L=32$ system the concentration $x$ oscillates with an amplitude $\delta x \simeq 0.10$; in the $L=256$ system we have $\delta x \simeq 0.02$, and in the $L=1024$ system the fluctuations are very small, $\delta x \simeq 0.004$. It is reasonable to conclude that for $s=2$ the correct solution is a stable fixed point, in perfect agreement with our theory.

In close connection with this last point, we have verified that for $s \geq 4$ the limit cycle obtained in the simulations is finite and stable, and independent of the size of the system up to size $L=1024$. For $s=3$ our ring kinetic theory predicts a fixed point $x=y=1 / 2$. However, simulations are here not conclusive, as systems of intermediate size $L=256$ show a limit cycle, but large systems do not reach any stationary behavior within available computer time. We conclude that the (inverse) Hopf bifurcation from a limit cycle to a fixed point at the level of the spatially averaged concentrations in a large enough system must occur between $s=2$ and $s=4$.

The comparison between ring kinetic theory and simulations has so far been restricted to 
the shape of the limit cycle. Figure 1 however does not give any information about the actual time evolution of the concentrations, or the period of oscillation around the limit cycle. In order to obtain this information, we have plotted in Fig. 3 the average concentration of the two species versus time, for both theory and simulations. Figure 3A and B show the concentration of particles of type $X$ and $Y$, respectively, for $s=10$. Simulation results are indicated by a solid line, while the ring kinetic theory is denoted by a dashed line. The amplitudes of the oscillation agree quite well, as we already knew from Fig. 1. There is however some deviation between theoretical and simulated periods, that causes the curves to become slightly out of phase. The difference between both oscillation periods is about $3 \%$. Figures $\mathrm{B} C$ and D show similar curves for $s=4$. Here the agreement is worse, and the difference in periods is about $11 \%$.

Figure 4 shows how the oscillation period - normalized by dividing by $s$ - depends on $s$. We have plotted the mean-field value of the period as a dashed line; the ring theory is denoted by circles, and simulation results by triangles. It was shown in Fig. 1 that ring kinetic theory predicts the shape of the limit cycle quite well down to $s \simeq 6$. It is therefore somewhat surprising that the mean-field prediction for the oscillation period, which is $s$-independent, is better than ring kinetic theory for all values of $s$. To resolve this issue it would be necessary to include higher order correlation functions in the theoretical description. This is clearly beyond the scope of the present paper. Furthermore, it can be seen that the approach to the mean-field period for large $s$ is slow, and even for $s=20$ there is a clear deviation of about $1 \%$. This effect is probably due to the particular choice of the transition rates, that are not able to maintain the local diffusive equilibrium even for high $s$ [2].

\section{DISCUSSION}

In this paper we have shown how a theory that takes into account equal-time pair correlations provides an excellent explanation of the large deviations from mean-field theory observed in diffusion-limited chemical reactions as modeled by lattice-gas automata (LGA). Our theory is a straightforward application of the general framework established in the papers of Ernst and coworkers [3,4]. It is not restricted to the Maginu model, but is applicable to any chemical reaction that can be modeled with an LGA.

It is in principle possible to include triplet and higher order correlations as well. However, the good agreement between theory and simulations indicates that the ring theory of section III] captures the essential physics in a quantitative way. Although the comparison between theory and simulations reported here is restricted to the domain of LGA's, we expect that mutatis mutandi the general concepts apply equally well to continuous systems.

We have focused on a particular two-species model exhibiting periodic oscillations of the average concentrations. Wu and Kapral [9] have studied a model with more complicated temporal behavior - period doubling bifurcations and a transition to a strange attractor, as model parameters are changed. They investigated the consequences of spatial fluctuations by means of computer simulations. It is an interesting question whether some of the features observed in that work can be explained using the theory presented in this paper. As a final remark we mention that our theory provides a more microscopic analogue of the Langevin equation method used in Ref. [10] to predict the magnitude of spatial density correlations. 


\section{ACKNOWLEDGMENTS}

It is a great pleasure to dedicate this paper to Matthieu Ernst on the occasion of his sixtieth birthday. Over the last decade, Matthieu has played an important role in developing a broad theoretical understanding of the behavior of lattice gas automata. Both authors have greatly enjoyed working with him, and appreciate his exceptional sense of responsibility, in particular when it comes to training young scientists.

We thank D. Dab and J. P. Boon for providing us with the table with the transition rates. R.B. acknowledges financial support from D.G.I.C.yT. (Spain), project PB94-0265. 


\section{REFERENCES}

[1] U. Frisch, D. d'Humières, B. Hasslacher, P. Lallemand, Y. Pomeau, and J.-P. Rivet, Complex Systems 1, 31 (1987) [reprinted in G. Doolen, ed., Lattice-Gas Methods for Partial Differential Equations (Addison-Wesley, Reading, Massachusetts, 1990)].

[2] J. P. Boon, D. Dab, R. Kapral, and A. Lawniczak, Phys. Rep. 273, 55 (1996).

[3] H.J. Bussemaker, M.H. Ernst, and J.W. Dufty, J. Stat. Phys. 78, 1521 (1995).

[4] M. H. Ernst and H. J. Bussemaker, J. Stat. Phys. 81, 515 (1995); R. Brito, H. J. Bussemaker, M. H. Ernst, and J. Matsui, Phys. Rev. E 52, 2657 (1995); H. J. Bussemaker, Phys. Rev. E 53, 1644 (1996); H. J. Bussemaker and M. H. Ernst, Phys. Rev. E 53, 5837 (1996).

[5] B. M. Boghosian and W. Taylor, Phys. Rev. E 52, 510 (1995).

[6] B. M. Boghosian and Taylor, J. Stat. Phys. 81, 295 (1995).

[7] D. Dab and J. P. Boon, and Y.-X. Li, Phys. Rev. Lett. 70, 1940 (1993).

[8] K. Maginu, Math. Biosci, 27, 17 (1975); J.Diff. Eqs., 31, 130 (1978).

[9] X.-G. Wu and R. Kapral, Phys. Rev. Lett. 70, 1940 (1993).

[10] J. R. Weimar, D. Dab, J. P. Boon, and S. Succi, Europhys. Lett. 20, 627 (1992). 


\section{FIGURES}

FIG. 1. Average concentrations $x$ and $y$ in the Maginu model for several values of the time scaling parameter: $s=4,6$, and 20. The outer dashed line corresponds to mean-field theory, given by Eqs. (化) and (5). Solid lines correspond to the ring kinetic theory of section [II]. The gray bands denote the result of computer simulations performed on square lattices of size $256 \times 256$; their width corresponds to the size of the fluctuations from cycle to cycle.

FIG. 2. Average concentration $x$ of species $X$ versus time $t$ for the Maginu model at $s=2$, and system size $32 \times 32,256 \times 256$, and $1024 \times 1024$, respectively. The bigger the system, the smaller the fluctuations.

FIG. 3. Concentration of species $X$ and $Y$ versus time $t$ for $s=10$ (figures A and B), and for $s=4$ (figures $\mathrm{C}$ and $\mathrm{D}$ ). Solid lines are the results of the computer simulations (in systems of size $256 \times 256$ ), while the dashed lines correspond to ring kinetic theory.

FIG. 4. Oscillation period as a function of $s$. The mean field value is indicated by a dashed line. Circles denote the ring kinetic theory prediction of section III. Triangles are the simulation values. Mean-field theory is here in better agreement with simulations than ring kinetic theory. 
FIGURE 1

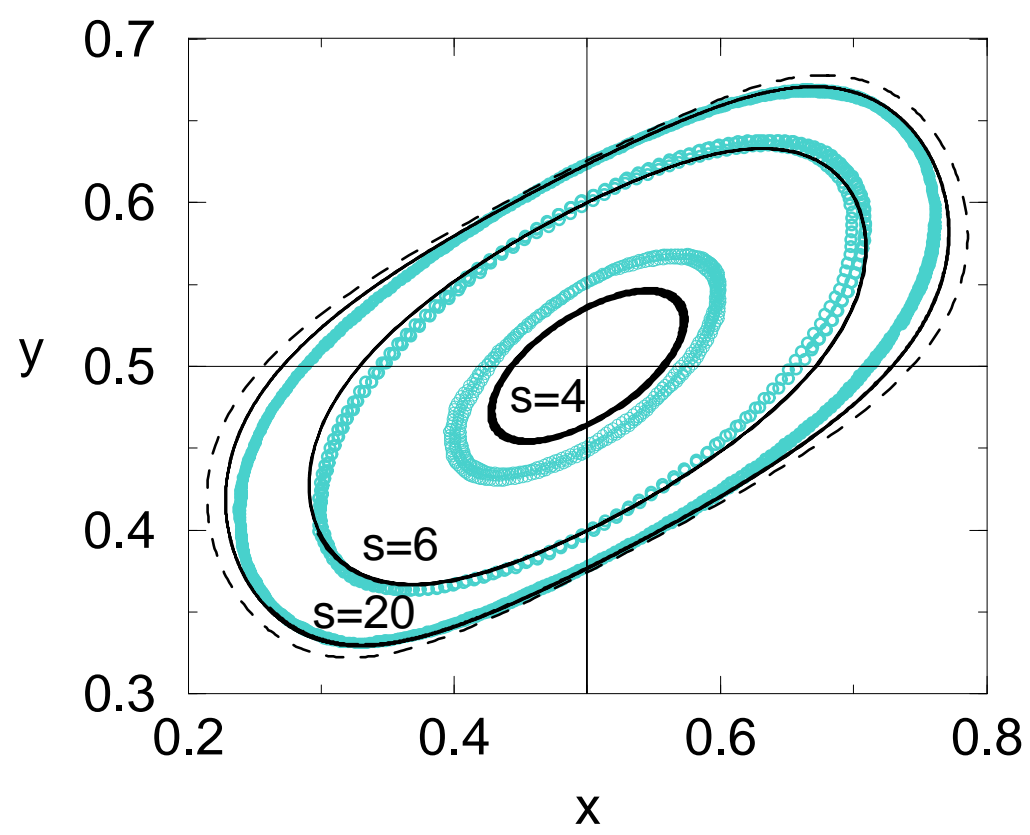

Title: Theory for Diffusion-Limited Oscillating Chemical Reactions Authors: H.J. Bussemaker and R. Brito 
FIGURE 2
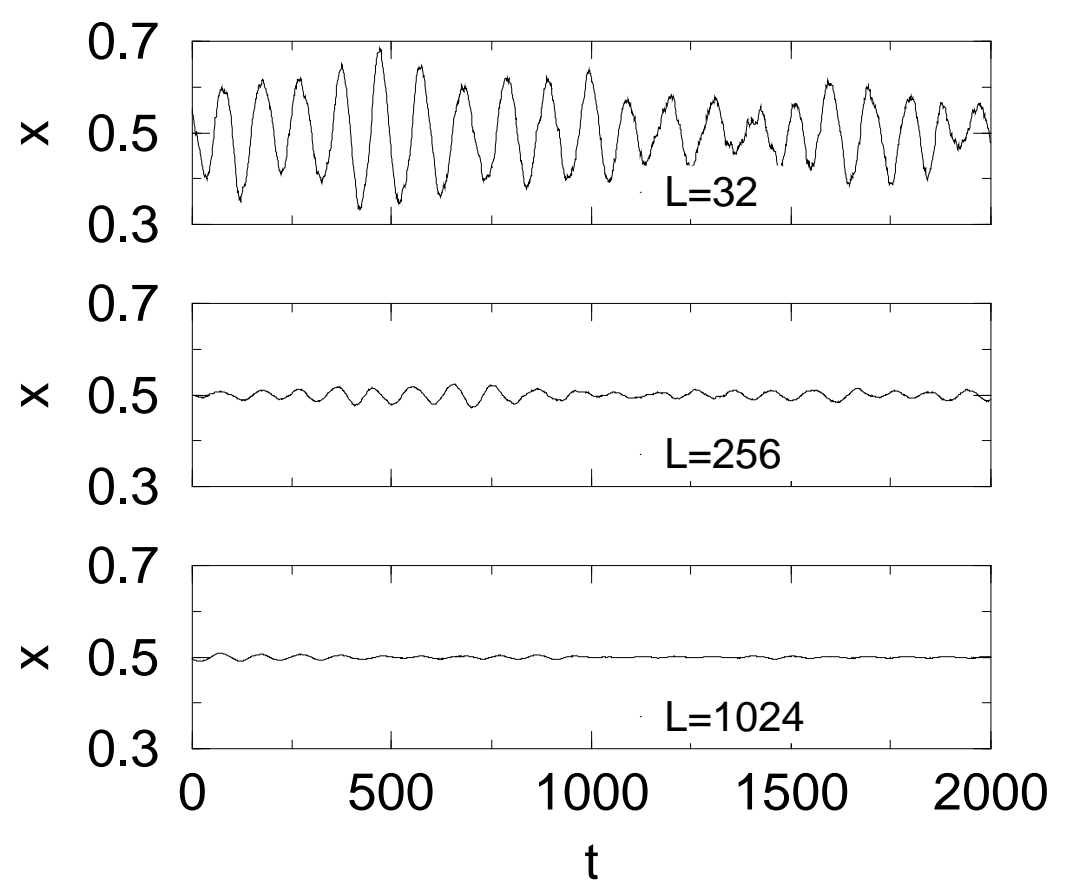

Title: Theory for Diffusion-Limited Oscillating Chemical Reactions Authors: H.J. Bussemaker and R. Brito 
FIGURE 3
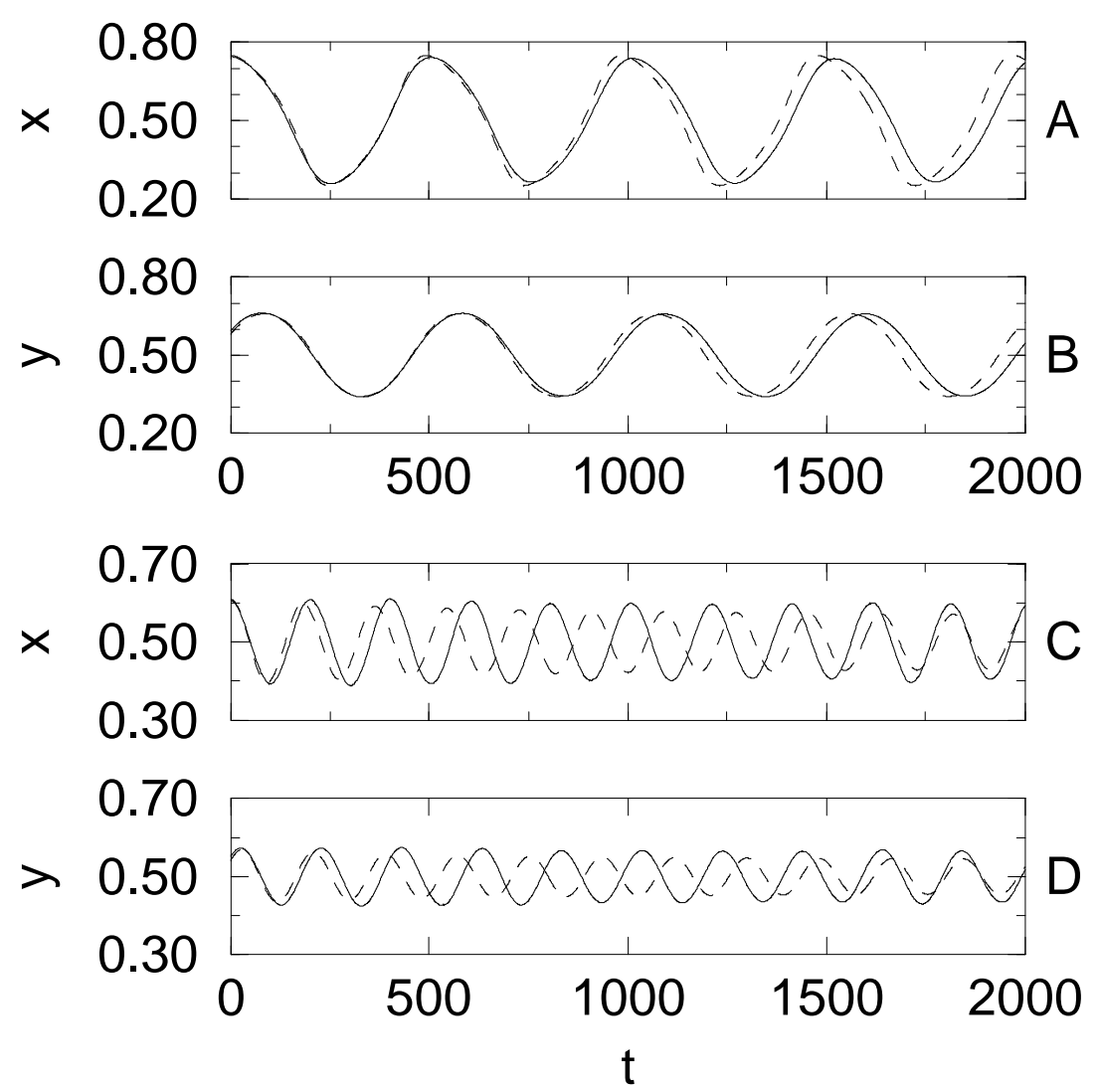

Title: Theory for Diffusion-Limited Oscillating Chemical Reactions Authors: H.J. Bussemaker and R. Brito 
FIGURE 4

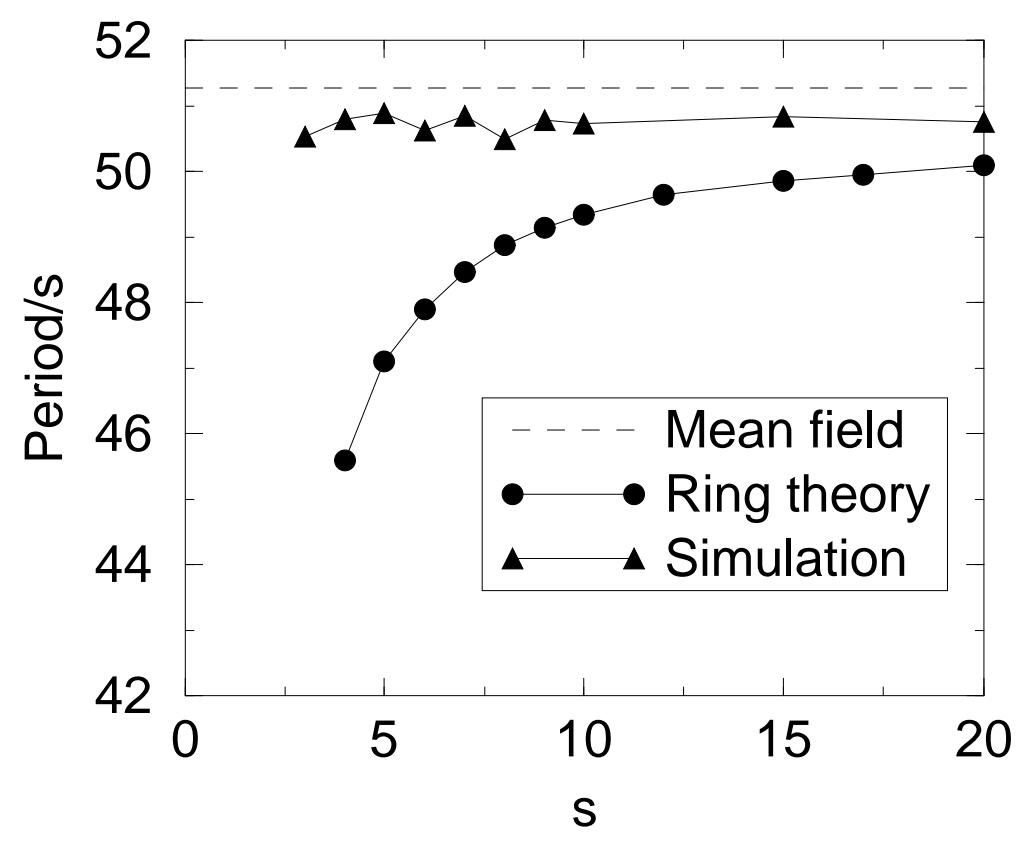

Title: Theory for Diffusion-Limited Oscillating Chemical Reactions Authors: H.J. Bussemaker and R. Brito 